Historic, Archive Document

Do not assume content reflects current scientific knowledge, policies, or practices. 
A984 Pro

U.S. Department of Agriculture

C 3 Animal and Plant Health Inspection Service

Program Aid No. 1430

\section{Rift Valley Fever}

A Mosquito-Borne Exotic Disease of Sheep, Cattle, and Humans

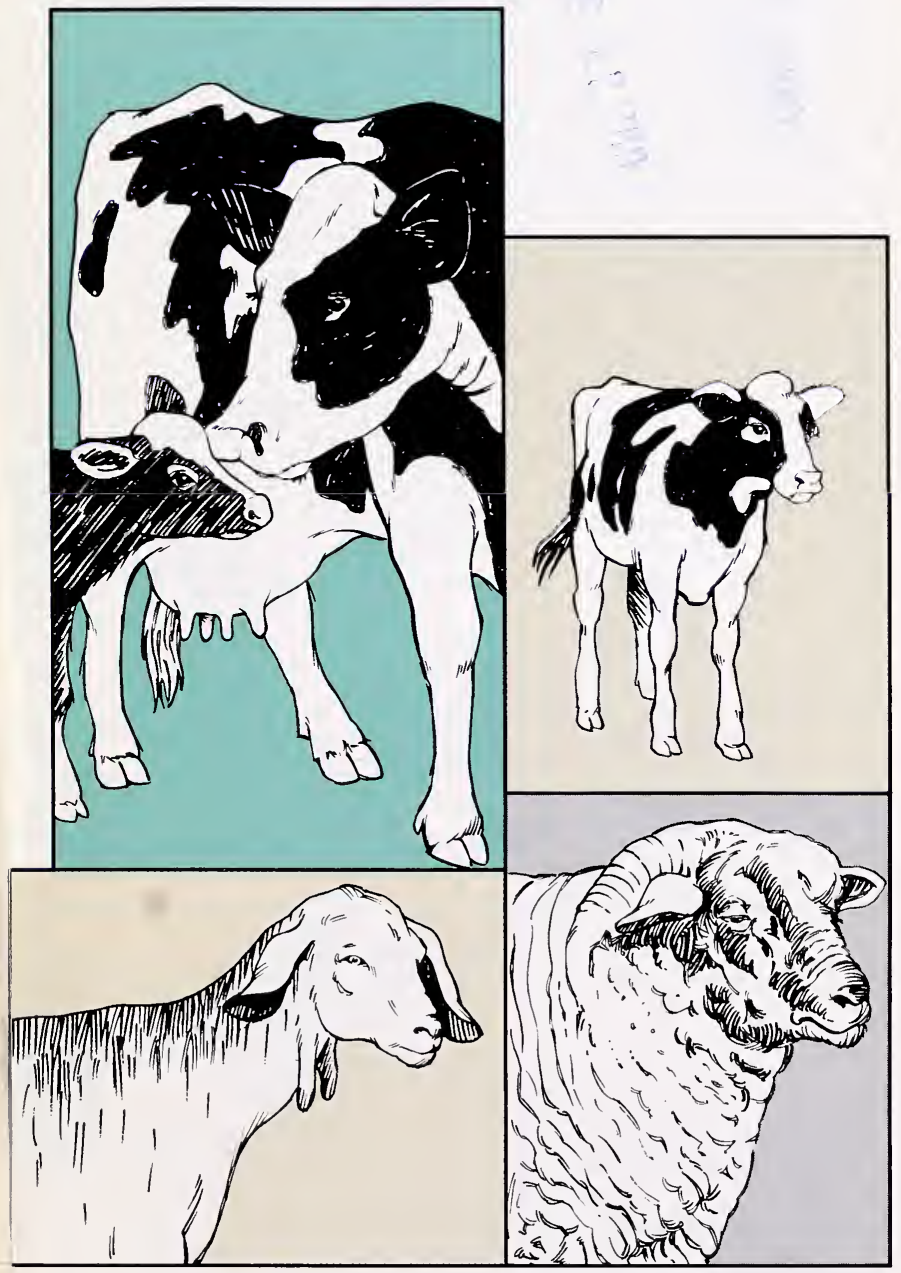




\section{Rift \\ Valley \\ Fever}

\section{A Mosquito-Borne Exotic Disease of Sheep, Cattle, and Humans}

Rift Valley fever is a viral disease spread by mosquitoes. It has not been reported outside Africa, but officials of the U.S. Department of Agriculture (USDA) see the disease as an ever-present threat to the United States.

USDA's Animal and Plant Health Inspection Service is alerting people to the dangers of this disease, in particular those people connected with the livestock industry and their veterinarians. They should know its clinical signs, especially the symptoms that differentiate this exotic disease from other, better known scourges of livestock.

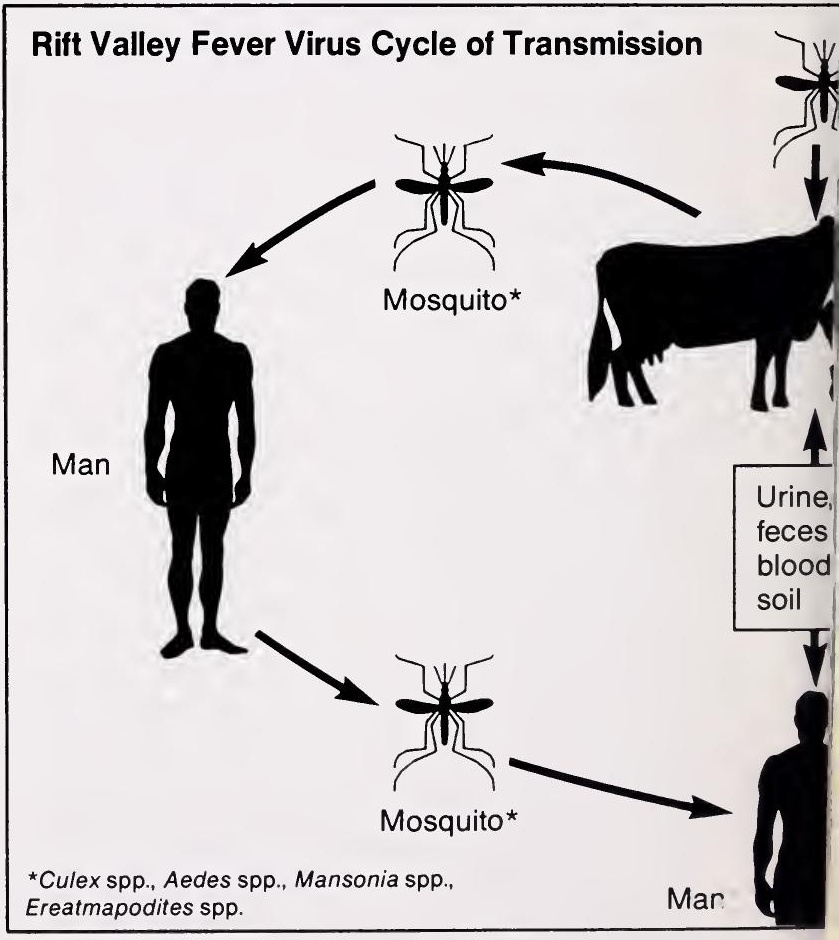

Rift Valley fever is transmitted between animals (and humans) by mosquitoes. Infection in humans also can occur through contact with blood or tissues. 


\section{Rift \\ Valley \\ Fever}

A Mosquito-Borne Exotic Disease

of Sheep, Cattle, and Humans

Rift Valley fever is a viral disease spread by mosquitoes. It has not been reported outside Africa, but officials of the U.S. Department of Agriculture (USDA) see the disease as an ever-present threat to the United States.

USDA's Animal and Plant Health Inspection Service is alerting people to the dangers of this disease, in particular those people connected with the livestock industry and their veterinarians. They should know its clinical signs, especially the symptoms that differentiate this exotic disease from other, better known scourges of livestock.

\section{History}

Rift Valley fever got its name from the place of its discovery in the Rift Valley of Kenya in 1931. From the first, those who experienced the disease have reported serious losses in lambs as well as infections in cattle, game animals, and humans.

Outbreaks of Rift Valley fever regularly recur in years of high rainfall when there is a large buildup in the number of mosquitoes that spread the virus. The disease is widespread in Africa (see map), but has not spread outside that continent.

An outbreak in Egypt in 1977 shows how disruptive the disease can be when it strikes a new area. The infection spread widely among domestic animals and humans, resulting in more than 700 riuman deaths.

\section{Signs}

Rift Valley fever causes high abortion rates in pregnant animals and high death losses in the young. The younger the animal, the higher the death rate. Mortality in newborn lambs, kids, and calves may exceed 95 percent.

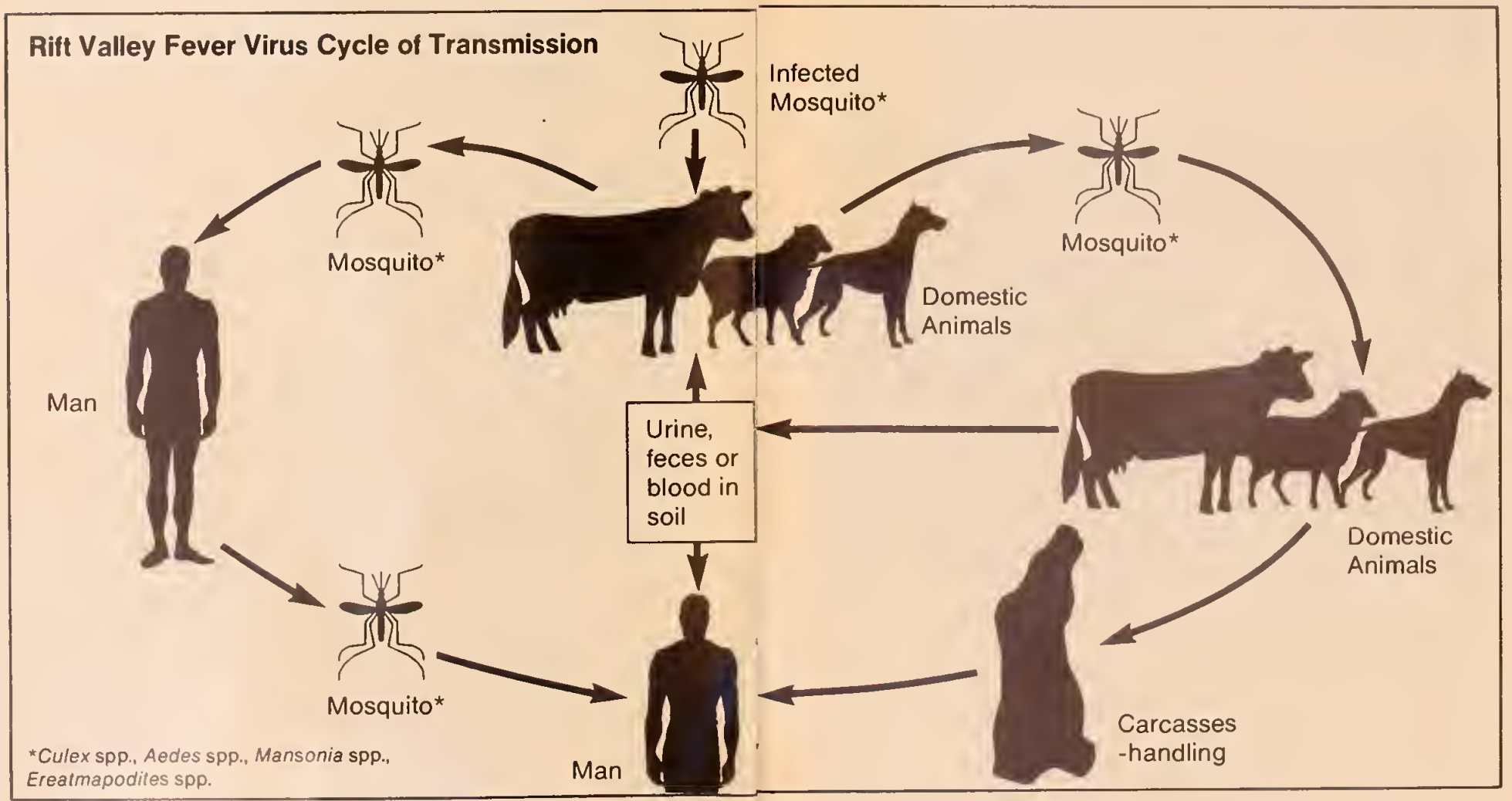

Rift Valley fever is transmitted between animals (and humans) by mosquitoes. Infection in humans also can occur through contact with blood or tissues. 


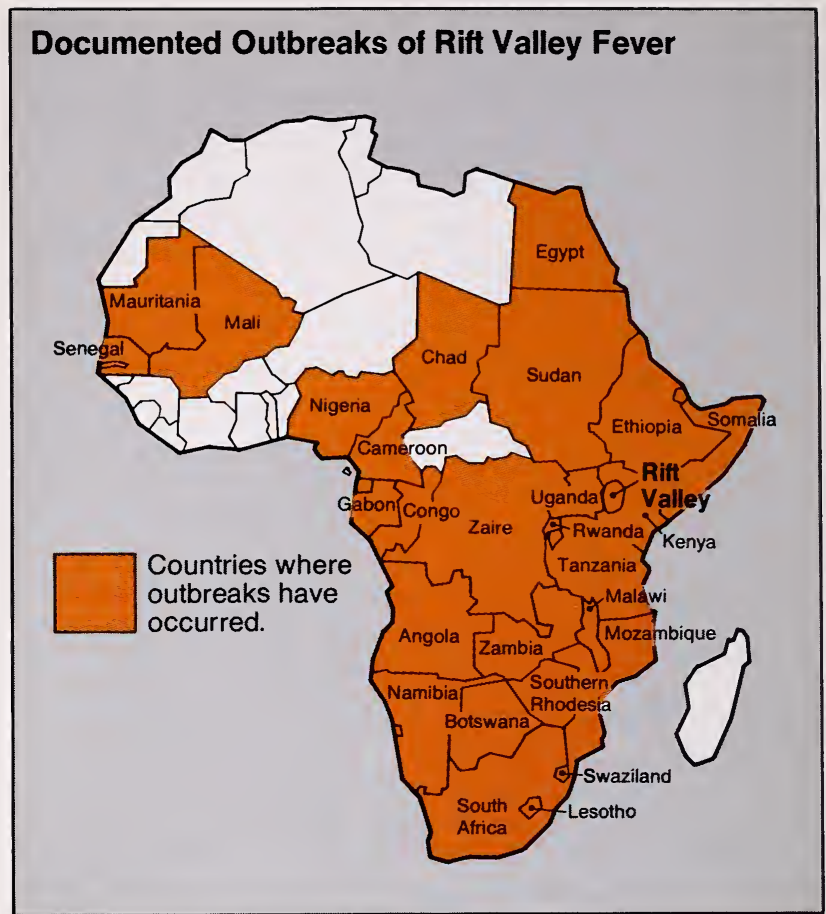

Rift Valley fever was first diagnosed along the Rift Valley in Kenya. Shaded areas indicate countries where outbreaks have occurred. None have been reported outside Africa.

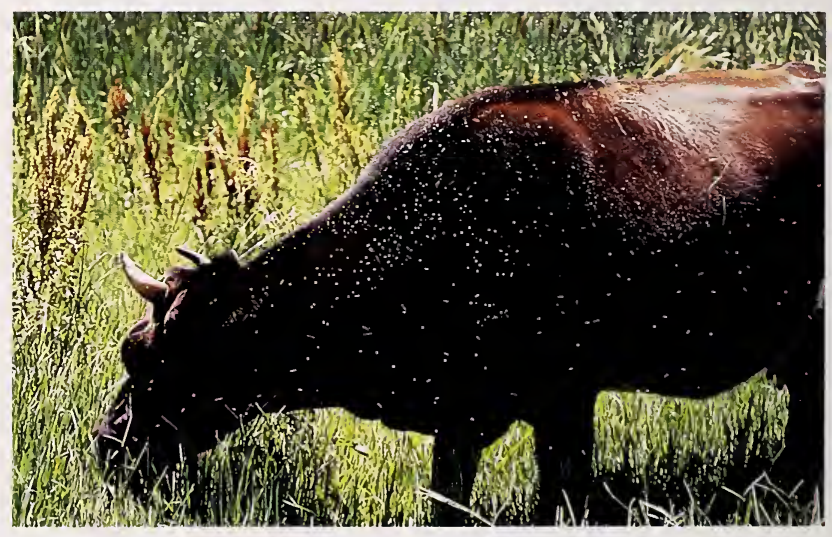

Jersey cow with a swarm of biting insects. Large swarms are needed to cause an outbreak. 
Rift Valley fever has its heaviest impact on sheep. Pregnant ewes abort in 95 to 100 percent of the cases. Signs develop suddenly, especially in young animals. In lambs under 1 week of age, the incubation period can be as short as 12 hours, followed by collapse and death within 16 hours. The incubation period in older lambs and adult sheep varies from 24 to 96 hours.

The usual progression of symptoms include a rapid rise in temperature, rapid pulse, muscle spasms, and unsteady gait. Pregnant ewes frequently abort. Infected animals may vomit and have a discharge from the nose.

Death usually takes place 24 to 48 hours after the onset of symptoms. In adult sheep, the death rate is usually about 20 percent, but it can be as high as 45 percent with some virulent types of virus. In some cases, no symptoms may be observed and animals may be found dead without previous signs of illness.

Rift Valley fever is less common in cattle than in sheep. However, in South Africa, cattle became infected on almost every farm where there were infected sheep.

The death rate in cattle varies from 10 to 30 percent. Infected cows often abort. Symptoms in adult cattle can be indefinite but usually include a period of high temperature, lack of appetite, excessive salivation, diarrhea, abdominal pain, rough hair coat, and cessation of milk production. Other symptoms can include erosions in the mouth, sore feet, and dead skin on the udder or scrotum.

Young goats with Rift Valley fever have signs similar to lambs. Adult goats are variably affected, but abortion rates can be high.

Humans who contract Rift Valley fever experience an abrupt rise in body temperature to 102-104 degrees Fahrenheit (39-40 degrees Centigrade) after a 4- to 6-day incubation period. Other symptoms resemble influenza and include chills, headache, muscular pain, weakness, vertigo, sensitivity to light, and a feeling of abdominal fullness, tenderness, and pain. Nausea and vomiting sometimes occur. The eyes also may be affected, causing eye pain and obscured vision. In serious cases, patients develop typical signs of hemorrhagic fever, such as bleeding from the nose, vomiting of blood, and passing of blood in the stools and urine. Death may result.

Patients who survive usually experience a rapid and complete recovery, although inflammation of some veins and eye damage occasionally persist for some time.

Recovered patients have at least a 20 -year immunity. 


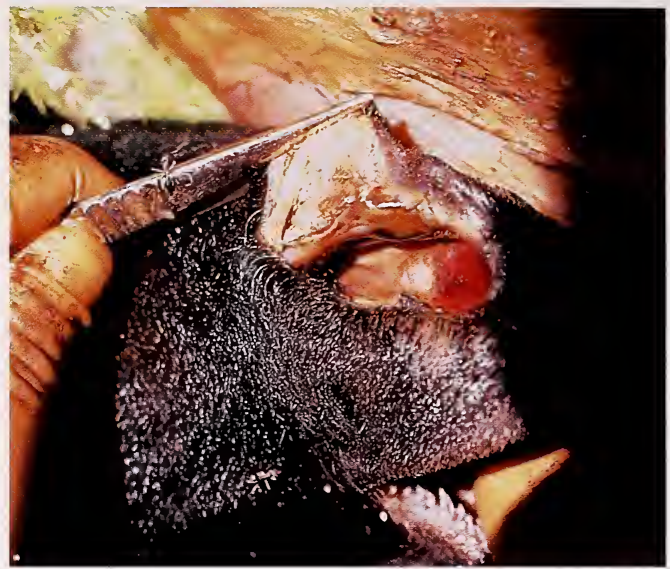

Focal hemorrhage and severe congestion in the nasal mucosa of a Karakul ewe.

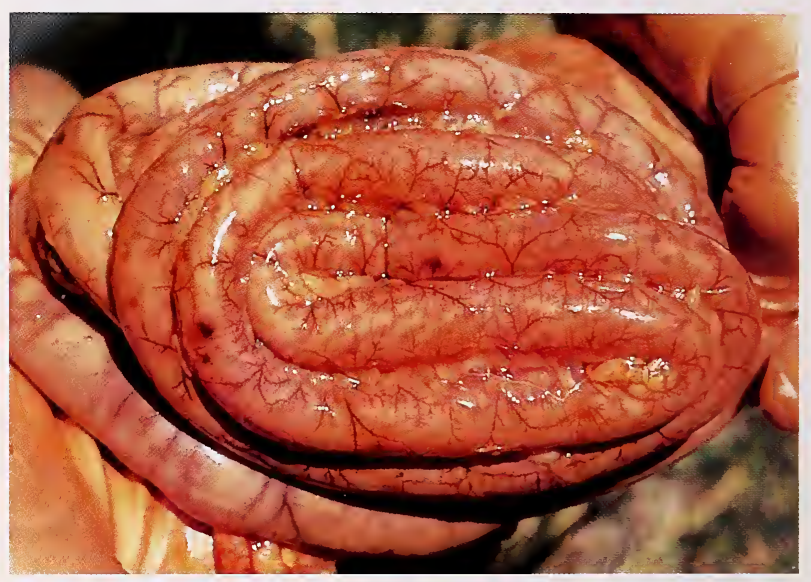

Colon of a Karakul ewe, showing pinpoint and focal hemorrhages in the serosa.

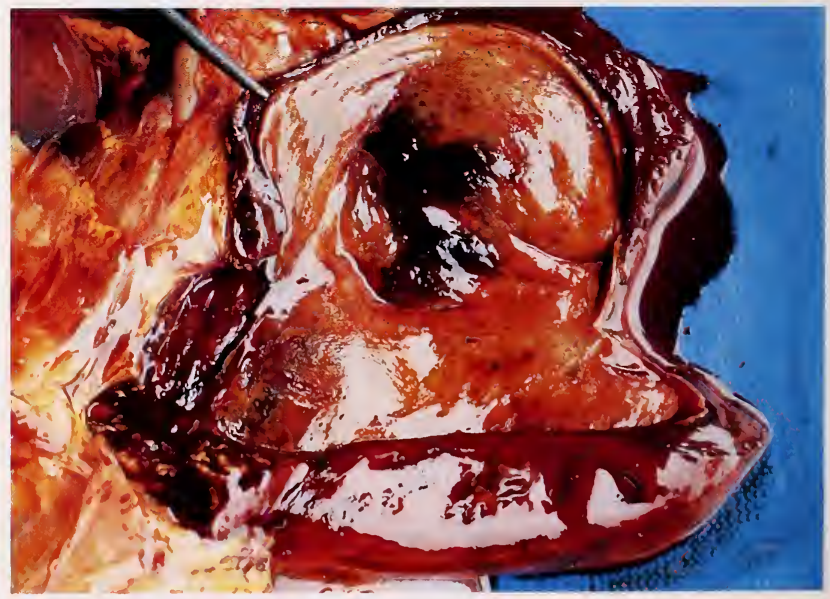

Incised gall bladder, showing severe hemorrhage between the serosal covering and mucosa. 


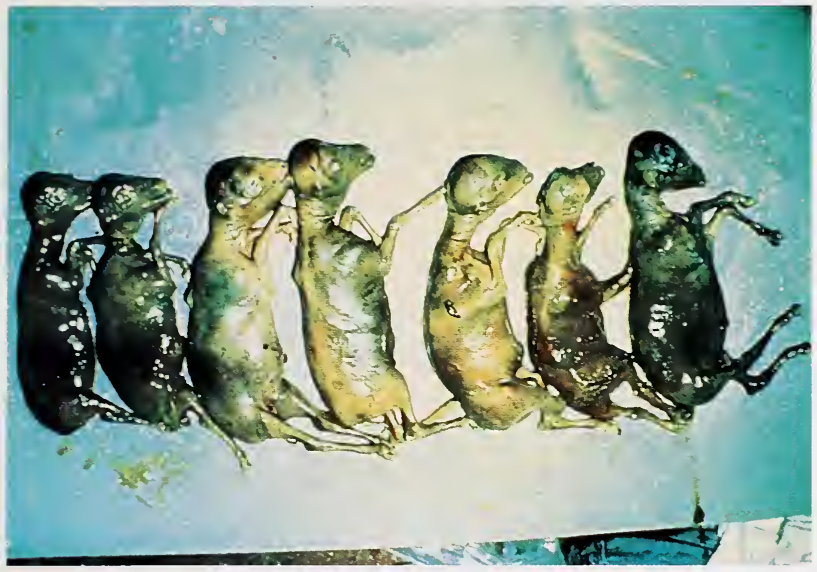

Between 95 and 100 percent of the ewes infected with Rift Valley fever abort. Fetuses often are hemorrhagic.

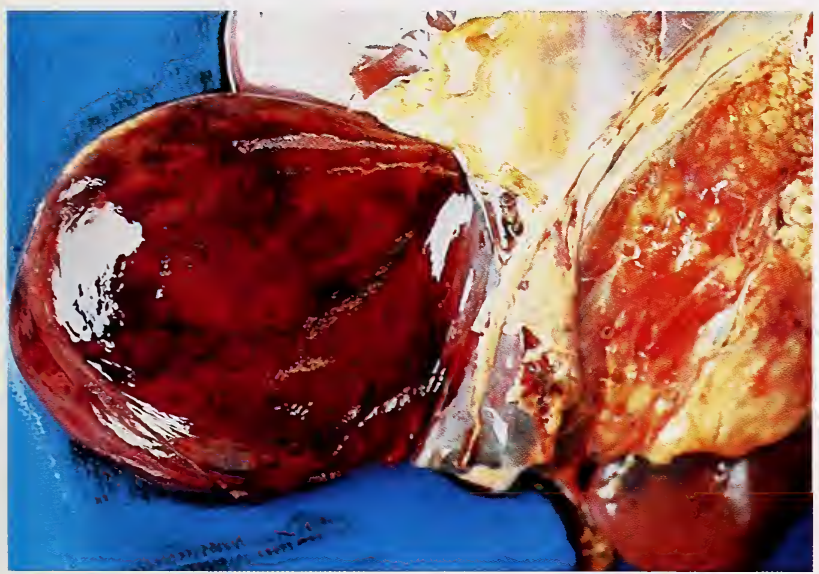

Liver from a mature cow with greatly enlarged gall bladder, probably due to congestion with blood.

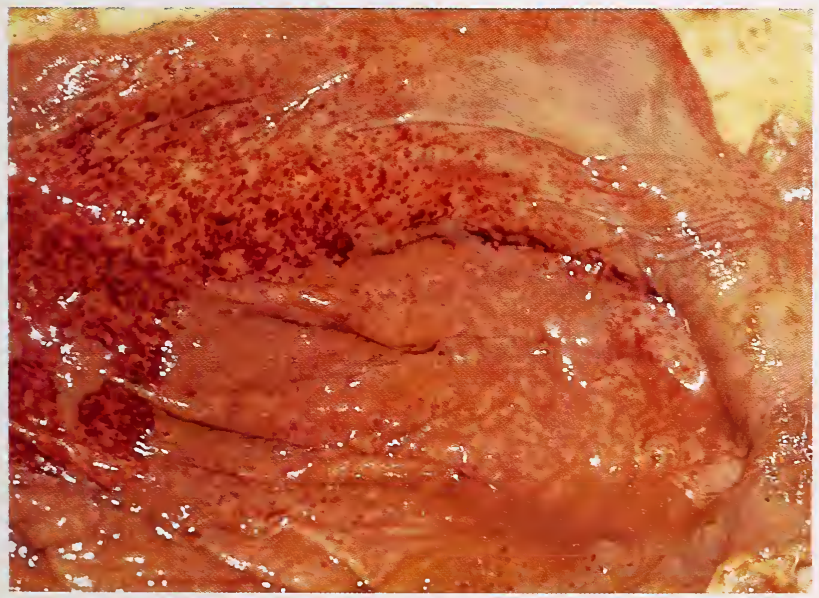

Abomasum of a Karakul ewe. Note edema and severe pinpoint and focal hemorrhages. 


\section{Post-Mortem Lesions}

In sheep, severe hemorrhages and edema can occur throughout the body in all organs and membranes.

One of the most striking post-mortem changes occurs in the liver and distinguishes Rift Valley fever from other diseases. The surface of the liver is mottled with areas of congestion, hemorrhage, and yellow areas of various sizes, caused by dead tissue. Dead tissue also may look red if it fills with blood; this may be quite pronounced in lambs, where most areas of some liver lobes may be involved. Other liver changes also are most severe in lambs.

The gall bladder often is swollen, congested, and hemorrhagic. Hemorrhages are common in the gastrointestinal tract, particularly the lining of the abomasum. Hemorrhages also can be found in the pancreas, kidneys, lungs, and lymph nodes.

The lungs often are swollen and have areas of hemorrhagic consolidation. The spleen can also be enlarged with focal areas of dead tissue. The lymph nodes can be enlarged and moist. In addition, there can be hemorrhages of the skin of the udder and flanks. Aborted fetal lambs often are hemorrhagic and show a marked hemothorax.

Post-mortem changes in cattle generally resemble those in sheep. Fresh hemorrhage is often found in the abomasum of cattle, accompanied by large visceral and serosal hemorrhages. Hemorrhages in the heart are more severe in cattle than in sheep.

Dead skin is common, especially on the udder and scrotum. The feet can become inflamed and the hooves may drop off.

Some investigators also have reported finding cattle with mouth lesions, typically including erosion of the lips, tongue, and mucous membranes. These investigators were undecided, however, whether these lesions were due solely to Rift Valley fever or possibly were evidence of concurrent infection with other viral diseases, like bluetongue or virus diarrhea.

\section{Confusion with Other Diseases}

Even an experienced eye finds it hard to differentiate Rift Valley fever from similar diseases. It resembles enterotoxemia, bluetongue, ephemeral fever, bruceilosis, vibriosis, and trichomoniasis. In fact, Nairobi sheep disease 


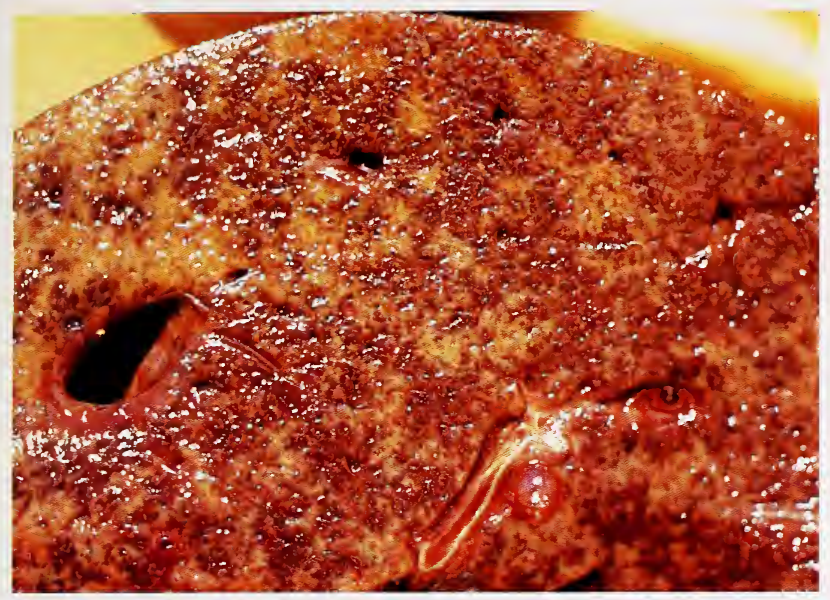

Multifocal necrosis in a lamb liver, a condition common in neonatal lambs, calves, and aborted fetuses.
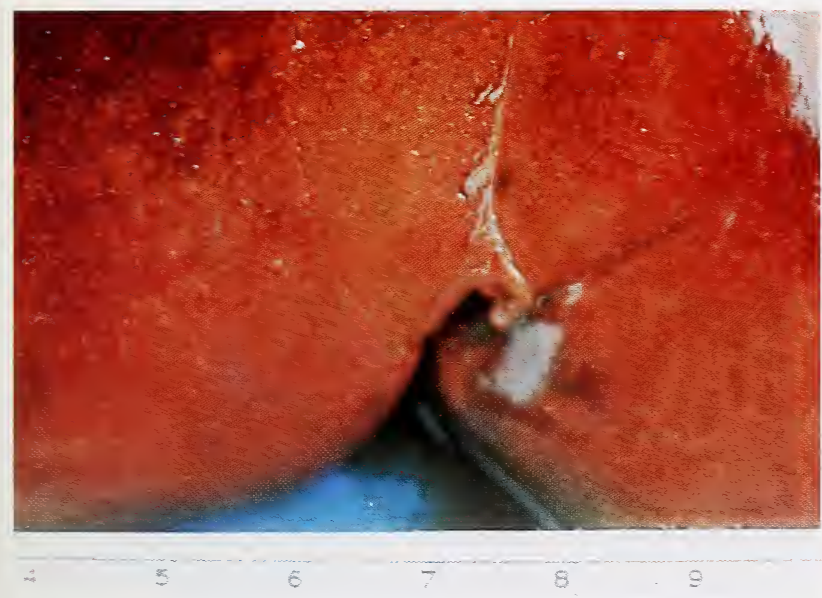

Liver section from a Karakul ewe with a more diffuse hemorrhagic appearance, caused by a confluence of focal lesions.

and ovine enzootic abortion should also be considered, since abortion often is the only sign of Rift Valley fever.

The only accurate method to confirm Rift Valley fever is to arrange a laboratory diagnosis. Submit tissue samples, especially of the liver, as well as blood samples, for analysis.

\section{How It Spreads}

Rift Valley fever is caused by an arbovirus of the Bunyaviridae family. It has many subtypes.

Mosquitoes appear to be the only natural means of transmitting the virus from animal to animal. Entomologists 
have isolated the virus from at least 16 species of mosquitoes.

Direct transmission between animals is rare; many researchers say it cannot occur. Even lambs nursing ewes with clinical symptoms remain uninfected. Occasional excretion of the virus in feces, urine, and milk is possible; however, there is no evidence that this plays a major role in transmission. The virus also can be found in the blood and other tissues from infected animals.

Direct human contact with the virus can result in infection. People can become infected directly through the skin or mucous membranes, by being punctured with infected needles, or by inhaling the virus. Workers who might be exposed to the virus should be immunized with human vaccine.

\section{Susceptible Species}

Rift Valley fever primarily affects sheep, cattle, and goats. The youngest animals are the most susceptible.

Humans, dogs, and cats also are susceptible, while horses and swine are resistant.

\section{Prevention and Control}

The policy of the U.S. Department of Agriculture toward exotic communicable diseases of livestock has two basic tenets: (1) Keep the disease out if at all possible; (2) if the disease breaches our defenses, eradicate it promptly. Keeping disease out of this country is more effective and much cheaper than fighting an outbreak here or "living with" the disease.

To prevent the entry of Rift Valley fever, USDA prohibits the entry of animal hosts from Africa or requires that they be isolated, prior to shipment, from insects that could carry the disease. Such restrictions apply to sheep, goats, cattle, and susceptible wild animals.

\section{What Can the U.S. Livestock Owner Do?}

All people connected with the livestock industry should know the clinical signs of Rift Valley fever. Early recognition of the symptoms and prompt notification of animal health officials are essential if eradication is to be achieved quickly. 
Livestock owners should observe their flocks or herds regularly for suspicious symptoms. Report symptoms immediately to a local veterinarian and State and Federal disease control officials.

Suspect Rift Valley fever if.

- A fatal disease occurs in lambs, kids, or calves following an increase in the number of mosquitoes in an area.

- The disease is milder in adult animals than in young animals.

- The disease causes abortion in pregnant sheep, goats, and cattle.

- The disease results in extensive necrotic changes of the liver.

- An influenza-like disease occurs in people working with infected animals or handling infected materials.

All programs and services are available to anyone without regard to race, color, sex, age, handicap, religion, or national origin.

Most photographs provided courtesy of the Armed Forces Institute of Pathology, Washington, D.C.

May 1989 
